\title{
Die Aufnahme partikulärer Nahrung bei Reniera sp. (Porifera)
}

\author{
Paul-Friedrich Langenbruch
}

\author{
Entwicklungsgeschichtliche Abteilung des Zoologischen Instituts der Universität Bonn; \\ Poppelsdorfer Schloß, D-5300 Bonn 1, Bundesrepublik Deutschland
}

\begin{abstract}
The uptake of particulate food in Reniera sp. (Porifera). The choanocyte chambers of the marine sponge Reniera sp. protrude with their curved outer surface free into the incurrent canals. The water is sucked into the chambers by cavities between the choanocytes. Particles up to 1 $\mu \mathrm{m}$ in diameter may enter the chambers with the water current. These particles are trapped on the outer surface of the choanocyte collars and are ingested by the choanocytes and processes of the pinacocyte epithelium of the incurrent canal system, which project into the chambers. Bigger particles are retained in the incurrent canals mainly on the outer surface of the choanocyte chambers. They are ingested by pinacocytes of the canal wall and transported to cells of the mesenchyme. The present investigation shows the great importance of the pinacocyte epithelium of the incurrent canal system for suspension feeding in Reniera sp.
\end{abstract}

\section{EINLEITUNG}

Schwämme sind filtrierende Organismen, die sich von partikulären und gelösten organischen Substanzen ernähren (Van Weel, 1949; Kilian, 1952, 1964; Claus et al., 1967; Rasmont, 1961, 1963; Reiswig, 1971, 1975a; Schmidt, 1970; Weissenfels, 1976; Wilkinson \& Garrone, 1980).

Dem Nahrungsgewinn dient bei Leucon-Schwämmen ein verzweigtes System einund ausführender Kanäle, die durch Choanocytenkammern miteinander verbunden sind. Die begeißelten Choanocyten erzeugen einen Wasserstrom, mit dem Nahrungspartikel in das Kanalsystem gelangen. Sie werden nach allgemeiner Auffassung zum großen Teil von den Choanocyten abfiltriert, phagocytiert und später an Zellen des Mesenchyms zu deren Ernährung weitergegeben. Von einigen Autoren wurde die Aufnahme von Partikeln auch durch Zellen der Kanalwände beobachtet (Van Weel, 1949, Kilian, 1952).

Die große Bedeutung der Choanocyten für die Ernährung der Zellen des Mesenchyms wird nur verständlich, wenn die Choanocytenkammern in direktem Kontakt zum Mesenchym stehen, was bisher (Sollas, 1888; Minchin, 1900; Hyman, 1940; Brien, 1973) für alle Leucon-Schwämme angenommen wurde. Bei Reniera sp. besitzen die Choanocytenkammern keinen Kontakt zum Mesenchym. Sie ragen vielmehr mit ihrer konvexen Außenfläche frei in die einführenden Kanäle (Langenbruch, 1983a). Es stellt sich daher die Frage, wie bei dieser Schwamm-Species die Ernährung des mesenchymatischen Gewebes gewährleistet wird. 
Der Bau der Choanocytenkammern und ihre Beziehung zum einführenden Kanalsystem wurden bisher kaum untersucht. Sie sind jedoch von entscheidender Bedeutung für das richtige Verständnis der Nahrungsaufnahme bei den Poriferen.

\section{MATERIAL UND METHODEN}

Von der Biologischen Anstalt Helgoland gelieferte Schwämme der Gattung Reniera (vermutlich Reniera cinerea [Grant]) wurden 9 Monate lang in einem 240-1-Seewasseraquarium bei $15^{\circ} \mathrm{C}$ und regelmäßiger Fütterung mit Liquizell (Fa. Dohse Aquaristik, Bonn), Liquifry marine (Liquifry Co. Ltd., Dorking, England) und diversem Trockenplankton gehalten. Zum Nachweis der Aufnahme partikulärer Substanzen wurde ein Teil der Schwämme eine halbe Stunde vor der Fixierung mit in Seewasser suspendierten Hefezellen (Saccharomyces cerevisiae) gefüttert. Die übrigen Schwämme erhielten vier Stunden vor der Fixierung Hefezellen.

Präparation für die Elektronenmikroskopie: (1) Fixierung: $2 \%$ Glutaraldehyd, $2 \%$ $\mathrm{OsO}_{4}$ und 5,5\% Rohrzucker in 0,05 M Na-Cacodylatpufferlösung, $\mathrm{pH} 7,0,4{ }^{\circ} \mathrm{C} 1 \mathrm{~h}$. (2) Wässerung: $6,9 \%$ Rohrzucker in 0,05 M Na-Cacodylatpufferlösung, pH 7,0,6 $610 \mathrm{~min}$. (3) Entkieselung: 5\% ige Flußsäure in Wässerungslösung (2), 2 h. (4) Wässerung: Wässerungslösung (2), $3 \times 10 \mathrm{~min}$. (5) Entwässerung: Athylalkoholreihe vom $15 \%$ igen bis zum abs. Alkohol in 7 Stufen, $20^{\circ} \mathrm{C}$, insgesamt $3^{1 / 2} \mathrm{~h}$. (6) Nachkontrastierung: $1 \%$ Phosphorwolframsäure und $1 \%$ Uranylacetat im 70igen Äthylalkohol der Entwässerungsreihe, $2 \mathrm{~h}$ bei $4{ }^{\circ} \mathrm{C}$. (7) Einbettung: Styrol-Methacrylat.

Die Präparation für die Rasterelektronenmikroskopie erfolgte nach einer von Weissenfels (1982) entwickelten, wiederholt beschriebenen Methode (Langenbruch, 1983a, 1984). In Styrol-Methacrylat eingebettete Gewebestücke von Reniera sp. wurden zunächst mikrotomiert. Anschließend wurden die nach der Mikrotomie im Polymerisat verbliebenen Reststücke durch Behandlung mit mehrfach gewechseltem Xylol vom Einbettungsmittel befreit, über die steigende Xylol-Amylacetatreihe in reines Amylacetat uiberführt und im Critical Point Dryer, Typ CPD 010, der Fa. Balzers getrocknet. Die Präparate wurden dann mit der Schnittfläche nach oben auf Halter für die Rasterelektronenmikroskopie aufgeklebt und im "kühlen" Sputter Coater der Fa. Polaron Equipment Ltd. mit Gold bedampft.

Mikrotomie: LKB-Ultrotome III. Transmissionselektronenmikroskopie: Zeiss EM 9 S-2 (Negativmaterial: Agfa Scientia). Rasterelektronenmikroskopie: Autoscan der Fa. Siemens (Negativmaterial: Agfa PAN 100 Professional).

Abb. 1. Median geschnittene Choanocytenkammer (ChK) von Reniera sp. zwischen einem ein- (eK) und einem ausführenden Kanal (aK). TEM-Aufnahme. Beiderseits der Apopyle (AP) sind Konuszellen (KZ) getroffen, die in Kontakt (-) zum Choanocytenepithel (Ch) und zu den Pinacocytenepithelien (PC) der beiden Kanalbereiche stehen. $\rightarrow$ präparationsbedingt ins Kammerlumen verschobene Choanocyten, PCF Pinacocytenfortsätze, M Mesenchym. $3700 \times$

Abb. 2. REM-Aufnahme eines angeschnittenen einführenden Kanals (eK) von Reniera sp. mit mehreren Choanocytenkammern (ChK). $\rightarrow$ Fortsätze der Choanocyten (Ch), - basale Kragenregion der Choanocyten, PC Pinacocyte, PCF Pinacocytenfortsätze, M Mesenchym. $2200 \times$ 

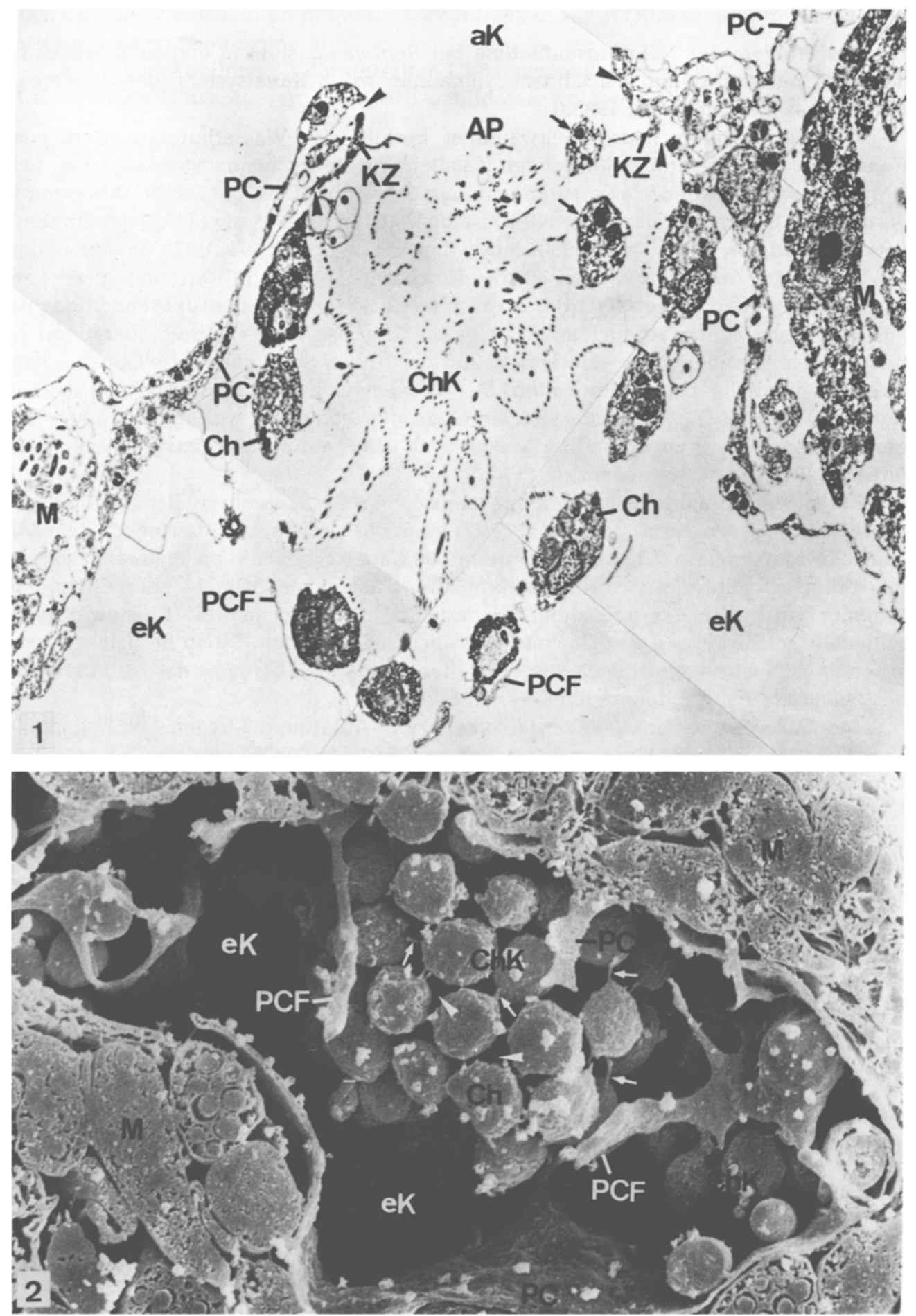


\section{ERGEBNISSE}

Das Problem der Nahrungsaufnahme bei Reniera sp. steht in engem Zusammenhang mit der Anordnung der Choanocytenkammern im Kanalsystem dieses Meeresschwammes (Langenbruch, 1983a).

Wie bei anderen Leucon-Schwämmen besteht das Wasserleitungssystem von Reniera sp. aus ein- und ausführenden Kanälen, die über Choanocytenkammern, den Antriebswerken für den Wasserstrom, miteinander verbunden sind. Die in Abbildung 1 dargestellte Choanocytenkammer (ChK) ist median durch die Apopyle (AP) geschnitten. Letztere wird von einem Ring aus Konuszellen (KZ) (Weissenfels, 1980) umgeben, der beiderseits der Apopyle getroffen ist. Die Konuszellen stehen in Verbindung $(-)$ zu den Pinacocytenepithelien (PC) des angrenzenden ein- (eK) und ausführenden Kanals $(\mathrm{aK})$. Zwei am rechten Apopylenrand gelegene Choanocyten $(\rightarrow)$ sind präparationsbedingt in das Kammerlumen verschoben. Auf der linken Seite haben die Choanocyten ihre ursprüngliche Lage beibehalten. Der Choanocytenverband grenzt hier an den Konuszell-Ring (KZ), von wo aus sich die Choanocytenkammer weit in das Lumen des einführenden Kanals vorwölbt. Ihre Außenfläche ist bis auf wenige, flache Pinacocytenfortsätze (PCF; Abb. 2) unbedeckt.

Bei angeschnittenen, vom Einbettungsmittel befreiten Gewebestücken von Reniera sp. (Abb. 2; vgl. "Material und Methoden") kann die fast unbedeckte Außenfläche der Choanocytenkammern (ChK) vom einführenden Kanalsystem (eK) her rasterelektronenmikroskopisch dargestellt werden. Zwischen den Choanocyten $(\mathrm{Ch})$ der beerenförmigen Kammer in der Mitte von Abbildung 2 befinden sich 1 bis 1,5 $\mu \mathrm{m}$ breite Spalten, die von schmalen Fortsätzen $(\rightarrow)$ der Choanocyten überbrückt werden. Durch die Lücken zwischen den Choanocyten sind an einigen Stellen die basalen Bereiche der aus Mikrovilli bestehenden Choanocytenkragen $(-$ ) zu erkennen.

Der Außenfläche der Choanocytenkammern in Abbildung 2 liegen vom Endopinacocytenepithel (PC) der Kanalwand ausgehende Fortsätze (PCF) auf. Die Choanocytenkammer in Abbildung 1 ist ebenfalls bereichsweise von Pinacocytenfortsätzen (PCF) bedeckt. Letztere sind in Ultradünnschnitten jedoch nur bei günstiger Schnittführung als Fortsätze des Endopinacocyten-Epithels zu identifizieren. Die Endopinacocyten überspannen auf diese Weise die Außenfläche der Choanocytenkammern und können sogar durch die Lücken zwischen den Choanocyten bis zu den Kragen im Kammerinnern vordringen (Abb. 3 und 4).

Das Untersuchungsmaterial wurde bis zur Fixierung in künstlichem Meerwasser gehalten, das durch Zugabe von diversem Plankton einen hohen Gehalt an Schwebstoffen und Bakterien aufwies. Diese Partikel werden durch die Dermalporen der Schwämme in das einführende Kanalsystem eingeschwemmt und im Wasserstrom bis zu

Abb. 3. TEM-Aufnahme einer Choanocytenkammer (ChK) von Reniera sp. Pinacocyten (PC) senden Fortsätze (-) ins Innere der Kammer aus, von denen einer ( - ) mehrere Partikel $(\rightarrow)$ umgreift. *Vakuole mit Partikeln, G Geißeln, K Kragen der Choanocyten (Ch), PCF Pinacocytenfortsatz, NV Nahrungsvakuole, h phagocytierte Hefezelle, H Hefezelle im einführenden Kanal (eK). $5200 \times$

Abb. 4. Teilbereich einer Choanocytenkammer von Reniera sp. TEM-Aufnahme. Ch Choanocyten, G Geißeln, K Kragen der Choanocyten, - ins Innere der Kammer ragender Pinacocyten (PC) Fortsatz $\rightarrow$ Phagocytose eines Partikels, V Vakuolen. $10100 \times$ 
den Choanocytenkammern mitgeführt. Dort können sie in Ultradünnschnitten nachgewiesen werden (Abb. 3 und 4 ).

Bei der in Abbildung 3 dargestellten Choanocytenkammer (ChK) erkennt man die Choanocyten $(\mathrm{Ch})$, die von Mikrovilli gebildeten Kragen $(\mathrm{K})$ und gelegentlich auch

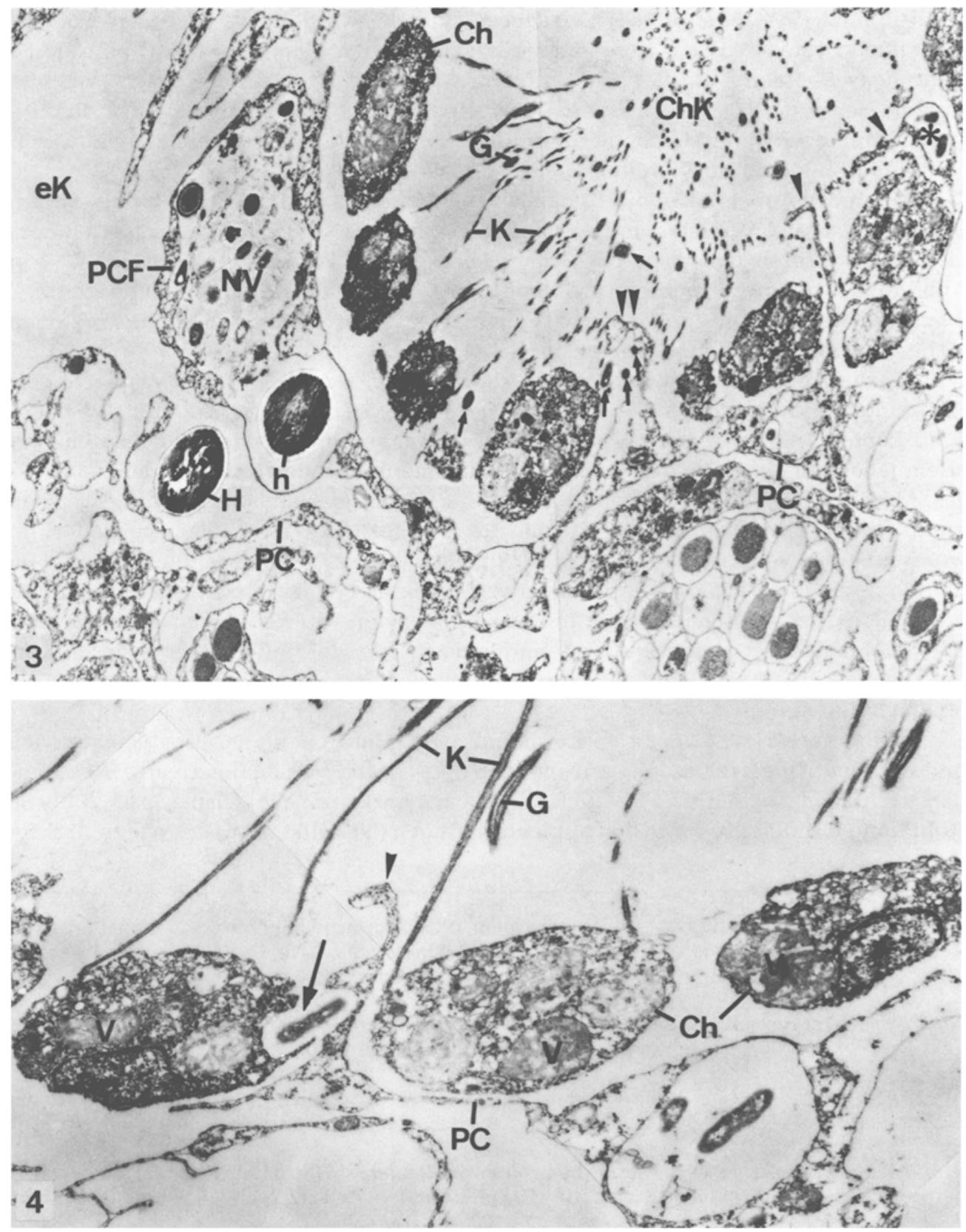


Geißeln (G). Der Kragenaußenseite liegen etwa $0,5 \mu \mathrm{m}$ große Partikel $(\rightarrow)$ an, die offensichtlich phagocytiert werden $(\rightarrow$ in Abb. 4). Auf eine rege Phagocytosetätigkeit der Choanocyten lassen auch zahlreiche Vakuolen (V in Abb. 4) mit unterschiedlich weit verdauter Nahrung schließen.

Am linken Rand der Abbildung 3 liegt ein einführender Kanal (eK), dessen Pinacocyten-Epithel (PC) unten an der Choanocytenkammer vorbeiführt. Der lange Fortsatz einer Pinacocyte (PCF) an der linken Außenfläche der Kammer enthält eine große Nahrungsvakuole (NV) mit phagocytiertem Material und einer Hefezelle (h). Eine weitere Hefezelle $(\mathrm{H})$ liegt frei im einführenden Kanal zwischen dem Pinacocytenfortsatz und einer anderen Endopinacocyte. In der Nähe der Choanocytenkammern trifft man in den Endopinacocyten immer auf viele Nahrungsvakuolen.

Die an der Außenfläche der Choanocytenkammern vorhandenen Endopinacocyten ( $\mathrm{PC}$ in $\mathrm{Abb} .3$ und 4) reichen mit Ausläufern (-) in die Choanocytenkammern hinein und phagocytieren dort angeschwemmte Partikel. Ein Pinacocyten-Fortsatz ( Abbildung 3 umgreift mehrere zwischen den Choanocytenkragen liegende Partikel. Zwei weitere Partikel sind in einer Vakuole (*) eines Pinacocyten-Fortsatzes oben rechts in Abbildung 3 zu erkennen.

Um die Aufnahme größerer Partikel darzustellen, wurden die Schwämme vor der Fixierung mit suspendierten Hefezellen (Saccharomyces cerevisiae) gefüttert. AnschlieBend konnten eingestrudelte Hefezellen im gesamten einführenden Kanalsystem, vor allem jedoch in der Nähe der Kragengeißelkammern, nachgewiesen werden.

Abbildung 5 stellt einen von Pinacocyten (PC) und Choanocyten ( $\mathrm{Ch}$ ) begrenzten einführenden Kanalbereich (eK) 30 min nach Hefezugabe dar. Zwei Hefezellen (h) wurden bereits von Endopinacocyten (PC) phagocytiert. Vier Hefezellen (H) liegen im einführenden Kanal (eK) vor einer Choanocytenkammer (ChK), ohne daß Anzeichen für eine Phagocytose erkennbar sind. In den Choanocyten von Reniera sp. konnten auch nach mehrstündiger Hefe-Fütterung nie phagocytierte Hefezellen nachgewiesen werden, obwohl die Choanocyten wie die Endopinacocyten direkt den einführenden Kanälen exponiert sind.

30 min nach Hefe-Zugabe treten phagocytierte Hefezellen nur in Endopinacocyten und in deren Fortsätzen an der Außenfläche der Choanocytenkammern auf. Vier Stunden nach Hefe-Zugabe sind sie auch in Mesenchymzellen nachweisbar (Abb. 6 bis 8). Abbildung 6 stellt eine durch den nukleolushaltigen (N) Zellkern (K) getroffene Archäo-

Abb. 5. Von Choanocyten (Ch) und Pinacocyten (PC) begrenzter einfuhrender Kanal (eK) von Reniera sp. TEM-Aufnahme. Die getroffenen Hefezellen liegen z. T. (h) in Vakuolen der Pinacocyten, z. T. (H) frei im Kanallumen. ChK Choanocytenkammer. $4000 \times$

Abb. 6. Archäocyte von Reniera sp. mit einer phagocytierten Hefezelle (h). TEM-Aufnahme. K Zellkern, N Nukleolus, V Vakuole. $10100 \times$

Abb. 7. Hefehaltige Mesenchymzelle von Reniera sp. TEM-Aufnahme. Die Hefezelle (h) ist von einer Membran der Mesenchymzelle und zwei zusätzlichen Membranen (-) umgeben. $10100 \times$

Abb. 8. TEM-Aufnahme zweier Mesenchymzellen von Reniera sp. Ein hefehaltiger (h), dünnwandiger $(-)$ Fortsatz ragt von der einen Zelle $(Z 1)$ in die andere Zelle $(Z$ 2) hinein. PP Pseudopodium. $10100 \times$ 

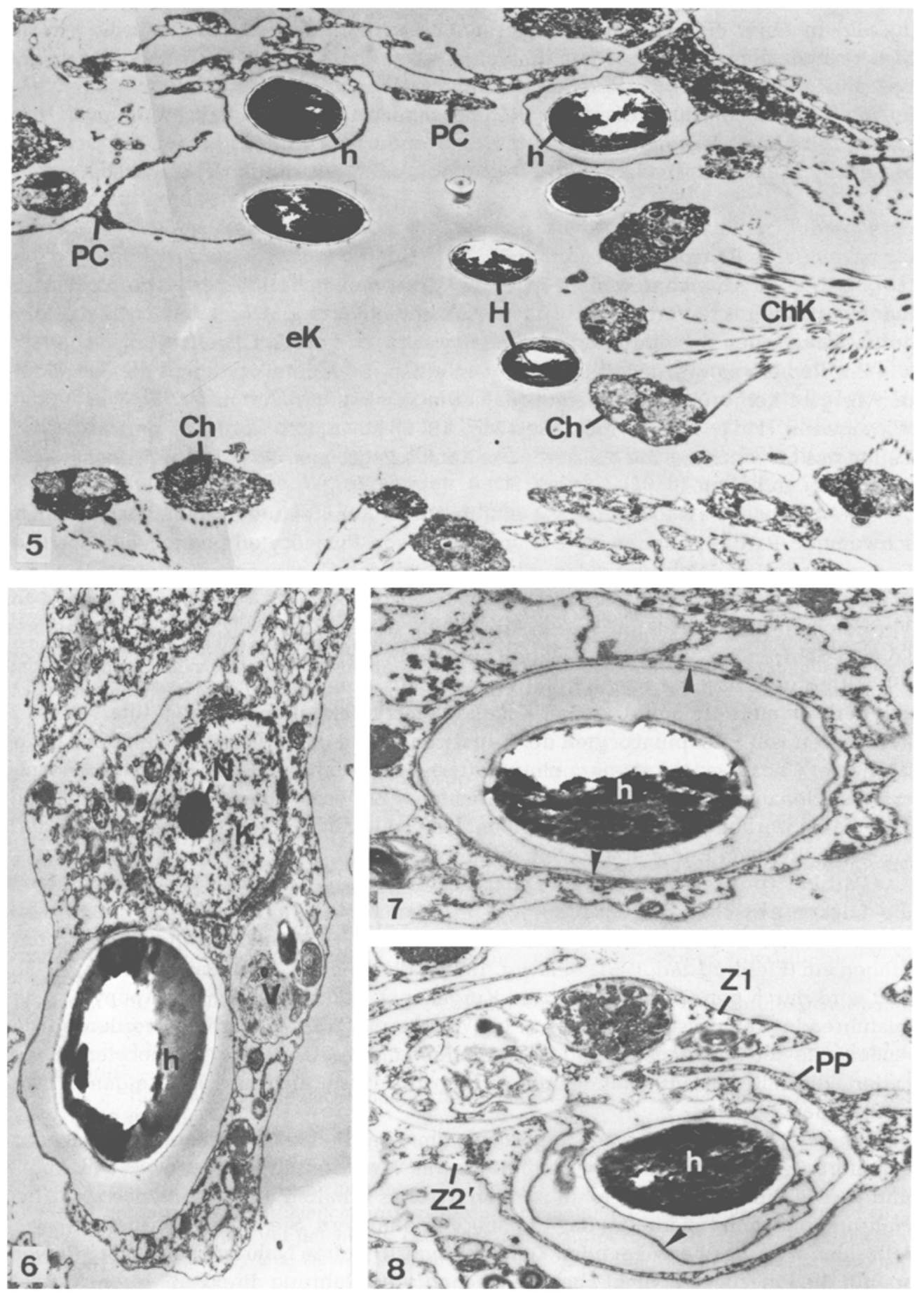
cyte dar. Sie enthält eine Vakuole (V) mit halbverdauter Nahrung und eine Hefezelle (h), die in einer einfachen Phagocytosevakuole liegt. Hin und wieder triff man in Mesenchymzellen auf Hefezellen, die von einer zusätzlichen, dünnen Zellhülle umgeben sind (Abb. 7 und 8.) Letztere kann mitunter nur aus zwei Membranen bestehen (in $\mathrm{Abb} .7$ und 8 ). In Abbildung 8 liegt eine besonders günstige Schnittführung vor. Man erkennt einen hefehaltigen (h) Fortsatz einer Mesenchymzelle (Z1), der in einer zweiten Mesenchymzelle (Z2) steckt, deren Pseudopodien (PP) den Zellfortsatz einschnüren.

\section{DISKUSSION}

Der Meeresschwamm Reniera sp. deckt einen großen Teil seines Nahrungsbedarfs durch partikuläres Material. Die primäre Aufnahme der eingestrudelten Partikel erfolgt durch Pinacocyten des einführenden Kanalsystems und durch Choanocyten.

Bei Reniera sp. werden alle Kanäle von einem Endopinacocytenepithel ausgekleidet. Es gibt keine Hinweise darauf, daß, wie von einigen Autoren (Van Weel, 1949; Wintermann, 1951; Kilian, 1952; Reiswig, 1971) für andere Poriferen berichtet wird, Zellen des Mesenchyms die einführenden Kanäle begrenzen oder sich in nennenswerter Zahl darin aufhalten.

Während der Passage durch die einführenden Kanäle an den Kanalwänden angeschwemmte Partikel können an Ort und Stelle von Pinacocyten phagocytiert werden. Der größte Teil der Nahrung gelangt jedoch bis zu den Choanocytenkammern. Die 1 bis $1,5 \mu \mathrm{m}$ weiten Einströmöffnungen der Choanocytenkammern von Reniera sp. sind im Vergleich zu den 3 bis $6 \mu \mathrm{m}$ großen Prosopylen in den Choanocytenkammern anderer Leucon-Schwämme (Reiswig, 1975b; Langenbruch, 1983b) relativ klein. Sie stellen Filterstrukturen dar, die alle Partikel von mehr als $1 \mu \mathrm{m}$ Durchmesser an der Außenfläche der Kammern im einführenden Kanalsystem zurückhalten. Die abgefilterten Partikel werden von Endopinacocyten der Kanalwände und deren Fortsätzen an der Außenfläche der Choanocytenkammern phagocytiert. Es ist damit zu rechnen, daß die Choanocyten an der zum einführenden Kanal gerichteten Zellbasis keine Nahrung aufnehmen. Jedenfalls werden dort angeschwemmte Hefezellen nie von Choanocyten, sondern ausschließlich von Endopinacocyten aufgenommen.

Partikel von weniger als $1 \mu \mathrm{m}$ Durchmesser gelangen mit dem Wasserstrom durch die Lücken zwischen den Choanocyten ins Kammerinnere. Das Wasser strömt dann durch Schlitze zwischen den Kragen-Mikrovilli (- in Abb. 2) in die Choanocytenkragen ein (Fjerdingstad, 1961; Reiswig, 1975a; Weissenfels, 1976; Langenbruch, 1983b) und wird durch den Geißelschlag zum Kragenapex und schließlich via Apopyle in das ausführende Kanalsystem befördert. Im Wasser mitgeführte Partikel werden an der Außenseite der Choanocytenkragen abgefangen und $\mathrm{z}$. $\mathrm{T}$. von den Choanocyten phagocytiert, die mit den aufgenommenen Substanzen vermutlich nur ihren eigenen Nahrungsbedarf decken.

An den Choanocytenkragen angeschwemmte Partikel können ebenfalls von Pinacocytenfortsätzen phagocytiert werden, die in die Kammern hineinragen ( - in Abb. 3 und 4). Die Pinacocyten des einführenden Systems erhalten auf diese Weise zusätzlich Nahrung aus dem Innenraum der Choanocytenkammern. Sie nehmen insgesamt zweifellos den weitaus überwiegenden Teil der eingestrudelten Nahrung auf. Da bei Reniera sp. nur die Pinacocyten, nicht aber die Choanocyten Nahrung direkt an Mesenchymzel- 
len weitergeben können, erscheint dies im Hinblick auf eine wirkungsvolle Ernährung des mesenchymatischen Gewebes vorteilhaft.

Johnston \& Hildemann (1982) wiesen bei dem Meeresschwamm Callyspongia diffusa (Ordnung Haplosclerida) an der Außenfläche der ebenfalls frei in die einführenden Kanäle ragenden Choanocytenkammern Zellenbereiche nach, bei denen es sich um Amöbocyten handeln soll. Bei Reniera sp. konnten in der vorliegenden Untersuchung entsprechende Zellenbereiche zweifellos als Fortsätze der Kanalwand-Pinacocyten identifiziert werden.

Die Nahrungsübermittlung von Pinacocyten zu Mesenchymzellen konnte nicht dokumentiert werden. Dieser Vorgang dürfte verhältnismäßig schnell ablaufen, so daß die Wahrscheinlichkeit, entsprechende Stadien zu treffen, außerordentlich gering ist. Phagocytierte Hefezellen liegen innerhalb der Pinacocyten immer in einer einfachen Phagocytose-Vakuole. In den Mesenchymzellen sind sie mitunter von einer zweiten, dünnen Zellhülle umgeben (Abb. 7). Die letztgenannte Beobachtung steht aller Wahrscheinlichkeit nach im Zusammenhang mit der Weitergabe bereits phagocytierter Hefezellen an andere Schwammzellen. Hierbei wird das phagocytierte Material in dünnwandige Zellfortsätze verlagert, die schließlich in die Empfängerzelle hineinragen (Abb. 8). Auf die gleiche Weise werden nach Willenz (1980) bei dem Süßwasserschwamm Ephydatia fluviatilis Latex-Partikel von Choanocyten an Archäocyten weitergegeben.

Danksagungen. Die Arbeit wurde durch Mittel der Deutschen Forschungsgemeinschaft gefördert. Herrn Prof. Dr. N. Weissenfels danke ich für zahlreiche Ratschläge und Anregungen. Für technische Assistenz danke ich Frau M. Geis, Frau I. Nüssle, Frau U. Müller und Frau B. Zarbock. Die rasterelektronenmikroskopische Aufnahme wurde am Max-Planck-Institut für Systemphysiologie in Dortmund angefertigt. Ich danke Herrn Prof. Dr. D. Lübbers, Herrn Dr. D. Schäfer und Frau M. Seiffert.

\section{LITERATUR}

Brien, P., 1973. Les Démosponges. In: Traité de zoologie. 3. Spongiaires. Ed. by P.-P. Grassé. Masson, Paris, 136-461.

Claus, G., Madri, P. P. \& Kunen, S. M., 1967. Removal of microbial pollutants from waste effluents by the redbeard sponge. - Nature, Lond. 216, 712-714.

Fjerdingstad, E. J., 1961. The ultrastructure of choanocyte collars in Spongilla lacustris (L). - Z. Zellforsch. mikrosk. Anat. 53, 645-657.

Hyman, L. H., 1940. The invertebrates. 1. Protozoa through Ctenophora. McGraw-Hill, New York, $726 \mathrm{pp}$.

Johnston, I. S. \& Hildemann, W. H., 1982. Cellular organisation in the marine Demosponge Callyspongia diffusa. - Mar. Biol. 67, 1-7.

Kilian, E. F., 1952. Wasserströmung und Nahrungsaufnahme beim Süßwasserschwamm Ephydatia fluviatilis. $-\mathrm{Z}$. vergl. Physiol. $34,407-447$.

Kilian, E. F., 1964. Zur Biologie der einheimischen Spongilliden. Ergebnisse und Probleme, - Zool. Beitr. 10, 85-159.

Langenbruch, P.-F., 1983a. Body structure of marine sponges. I. Arrangement of the flagellated chambers in the canal system of Reniera sp. - Mar. Biol. 75, 319-325.

Langenbruch, P.-F, 1983b. Untersuchungen zum Körperbau von Meeresschwämmen. II. Das Wasserleitungssystem von Halichondria panicea. - Helgoländer Meeresunters. 36, 337-346.

Langenbruch, P.-F., 1984. Vergleichende rasterelektronenmikroskopische Darstellung der Gemmulaschalen von Ephydatia fluviatilis, E. muelleri und Spongilla fragilis (Porifera). - Zoomorphology 104, 79-85. 
Minchin, E. A., 1900. Sponges. In: A treatise on zoology. 2. The Porifera and Coelenterata. Ed. by E. R. Lankester. Black, London, $178 \mathrm{pp}$.

Rasmont, R., 1961. Une technique de culture des éponges d'eau douce en milieu controlé. - Annls Soc. r. zool. Belg. 91, 147-155.

Rasmont, R., 1963. Le rôle de la taille et de la nutrition dans le déterminisme de la gemmulation chez les Spongillides. - Devl Biol. 8, 243-271.

Reiswig, H. M. 1971. Particle feeding in natural populations of three marine Demosponges. - Biol. Bull. mar. biol. Lab., Woods Hole 141, 568-591.

Reiswig. H. M., 1975a. Bacteria as food for temperate-water marine sponges. - Can. J. Zool. 53, $582-589$.

Reiswig, H. M., 1975b. The aquiferous systems of three marine Demospongiae. - J. Morph. 145, 493-502.

Schmidt, J., 1970. Phagocytose et pinocytose chez les Spongillidae. - Z. vergl. Physiol. 66, 398-420.

Sollas, W. J., 1888. Report on the Tetractinellida collected by H. M. S. "Challenger", during the years 1873-1876. - Rep. scient. Results Voyage HMS Challenger (Zool.) 25, 1-458.

Van Weel, P. B., 1949. On the physiology of the tropical freshwater sponge Spongilla proliferens Annand. I. Ingestion, digestion and excretion. - Physiol. comp. oecol. 1, 110-128.

Weissenfels, N., 1976. Bau und Funktion des Süßwasserschwamms Ephydatia fluviatilis L. (Porifera). III. Nahrungsaufnahme, Verdauung und Defäkation. - Zoomorphologie 85, 73-88,

Weissenfels, N., 1980. Bau und Funktion des Süßwasserschwamms Ephydatia fluviatilis L. (Porifera). VII. Die Porocyten. - Zoomorphologie, 95, 27-40.

Weissenfels, N., 1982. Rasterelektronenmikroskopische Histologie von spongiösem Material. Microsc. Acta $85,345-350$.

Wilkinson, C. \& Garrone, R., 1980. Nutrition of marine sponges. Involvement of symbiotic bacteria in the uptake of dissolved carbon. In: Nutrition in the lower Metazoa. Ed. by D. C. Smith \& Y. Tiffon. Pergamon Press, Oxford, 157-161.

Willenz, P., 1980. Kinetic and morphological aspects of particle ingestion by the freshwater sponge Ephydatia fluviatilis L. In: Nutrition in the lower metazoa. Ed. by D. C. Smith \& Y. Tiffon. Pergamon Press, Oxford, 163-178.

Wintermann, G., 1951. Entwicklungsphysiologische Untersuchungen an Süßwasserschwämmen. Zool. Jb. (Anat. Ontogenie Tiere) 71, 427-486. 